Supporting Information for

Phase Behavior of Phasmidic Mesogen-Jacketed Liquid Crystalline Polymers Displaying Chain Bundling

Jun-Feng Zheng,,*, Tao Tang ${ }^{1}$, Lin-Lin Ding ${ }^{1}$, Peng Xu${ }^{1}$, Rui Zhang, ${ }^{1, *}$, Dong-Lai Peng ${ }^{3}$, Shuang Yang ${ }^{2}$, Er-Qiang Chen,*

${ }^{1}$ Department of Applied Chemistry, College of Chemical Engineering, Nanjing Forestry University, Nanjing 210037, China

${ }^{2}$ Beijing National Laboratory for Molecular Sciences, Key Laboratory of Polymer Chemistry and Physics of the Ministry of Education, College of Chemistry, Peking University, Beijing 100871, China

${ }^{3}$ School of Material \& Chemical Engineering, Zhengzhou University of Light Industry, Zhengzhou 450001, P.R. China

Corresponding authors. E-mail: zjfphd@163.com (J.F.Z.); eqchen@pku.edu.cn (E.Q.C.); zhangrui@njfu.edu.cn (R.Z.).

\title{
Contents:
}

1. Materials and Characterization Techniques ...........................................................2

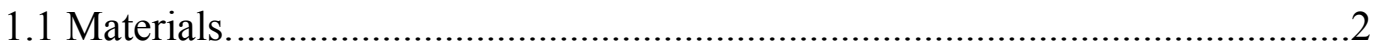

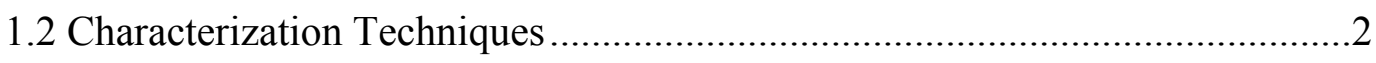

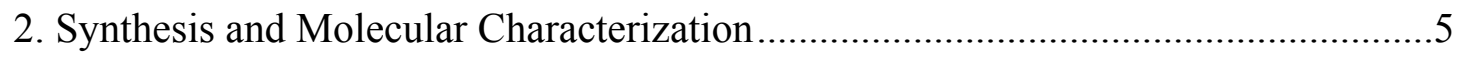

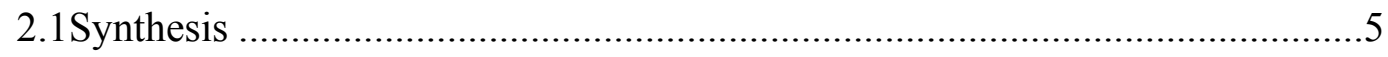

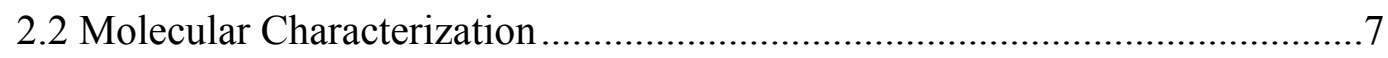

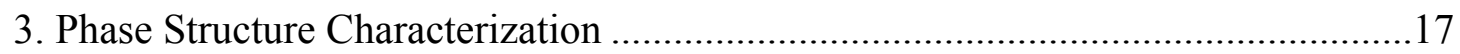

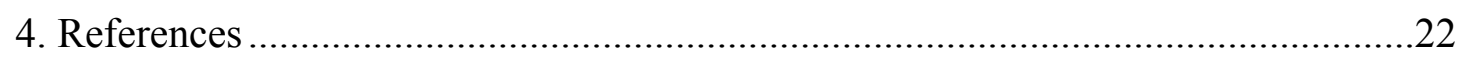




\section{Materials and Characterization Techniques}

\subsection{Materials.}

Chlorobenzene $\left(\mathrm{C}_{6} \mathrm{H}_{5} \mathrm{Cl}\right)$ was purified by sequentially washing with concentrated sulfuric and water, dried over $\mathrm{CaH}_{2}$, and then distilled. Benzoyl peroxide (BPO) was purified by recrystallized with chloroform-methanol mixture. Dichloromethane (DCM) was distilled over $\mathrm{CaH}_{2}$. Grubbs catalyst II and cis-5-Norbornene-exo-2,3-dicarboxylic anhydride were purchased from Sigma-Aldrich. All other reagents were obtained from commercial sources and used as received unless otherwise noted.

\subsection{Characterization Techniques}

NMR. ${ }^{1} \mathrm{H}$ NMR and ${ }^{13} \mathrm{C}$ NMR spectra were recorded on a Bruker Avance III600 spectrometer at room temperature using tetramethylsilane (TMS) as the internal standard. ${ }^{1} \mathrm{H}$ NMR chemical shifts are reported in parts per million (ppm) relative to solvent (chloroform, $7.27 \mathrm{ppm}$; dimethyl sulfoxide, $2.50 \mathrm{ppm}$ ). ${ }^{13} \mathrm{C}$ NMR chemical shifts are reported in ppm, and referenced to solvent (chloroform, $77.0 \mathrm{ppm}$ ).

GPC. GPC was carried out on a Waters 515 GPC instrument using THF as an eluent at $35{ }^{\circ} \mathrm{C}$. The GPC calibration curve was obtained with linear PS standards. Flow rate of THF was $1.0 \mathrm{ml} / \mathrm{min}$. Samples were diluted in $0.001-0.005 \mathrm{wt} \%$ by THF and filtered through $0.20 \mu \mathrm{m}$ PTFE filter before injection into the GPC.

POM. Birefringence properties of samples were evaluated by polarized optical 
microscopy (POM, Nikon LV100 POL) coupled with a Mettler hot stage (FP82HT). To sufficiently eliminate the thermal history effect and to obtain characteristic birefringence textures, images were collected during slow cooling processes $\left(1{ }^{\circ} \mathrm{C} / \mathrm{min}\right)$ from high temperatures such as isotropic temperatures.

DSC. Thermal transitions of samples were studied using differential scanning calorimetry (DSC) on a TA Q100 calorimeter in the temperature range of $-20 \sim 300{ }^{\circ} \mathrm{C}$. The temperature and heat flow were calibrated with benzoic acid and indium, respectively. The samples were encapsulated in hermetically sealed aluminum pans, with a typical sample weight of $\sim 4 \mathrm{mg}$. To eliminate thermal history, samples were first heated to high temperatures such as their isotropic temperatures. Then the experiments were conducted on the subsequent cooling and heating scans with a rate of $10{ }^{\circ} \mathrm{C} / \mathrm{min}$ under a nitrogen atmosphere.

1D XRD. One-dimensional (1D) X-ray diffraction (XRD) experiments were performed with a high-flux SAXS instrument (SAXSess, Anton Paar) equipped with Kratky block-collimation system. The diffraction of both small- and wide-angle can be simultaneously recorded on an imaging-plate (IP) with a pixel size of $42.3 \times 42.3$ $\mu \mathrm{m}$ which extended to high-angle range (the q range covered by the IP was from 0.06 to $29 \mathrm{~nm}^{-1}, q=4 \pi(\sin \theta) / \lambda$ where $\lambda$ is the wavelength of $0.1542 \mathrm{~nm}$ and $2 \theta$ is the scattering angle). The diffraction peak positions were calibrated with silicon powder for wide-angle region and silver behenate for small-angle region, respectively.

Sample Preparation for 1D XRD Measurement. A powder sample ( $\sim 15 \mathrm{mg})$ on a glass slide was heated to high-temperature by a Mettler hot stage and then cooled 
at $1{ }^{\circ} \mathrm{C} / \mathrm{min}$ to room temperature (RT), affording a $\sim 3$-mm-thick film. The film was peeled off by a razor blade and sandwiched between two aluminum foil sheets and exposed to X-ray for $20 \mathrm{~min}$. For variable-temperature 1D XRD experiments, data of small molecules were collected during cooling processes, and those of polymers were collected during heating processes.

2D XRD. Two-dimensional (2D) XRD patterns of the oriented samples were recorded by employing a Bruker D8 Discover diffractometer with a Vantec 500 detector which was also calibrated with silicon powder and silver behenate. To perform 2D XRD experiments, the macroscopically oriented samples were prepared by mechanical shear on a solid substrate at temperatures just above LC isotropization temperatures. The 2D XRD patterns were recorded in transmission mode, with the X-ray incident beam aligned perpendicular and parallel to the shear direction. The background scattering was recorded and then subtracted.

Preparation of Oriented Films for 2D XRD Measurement. Powder samples ( $\sim 15 \mathrm{mg}$ ) on glass slides were heated to high-temperatures and then cooled to shearing temperatures followed by annealing for one hour. The shearing temperatures of MS- $n$, PNB- $n$, and PE- $n(n=8,12)$ are $\sim 90, \sim 150$, and $\sim 250{ }^{\circ} \mathrm{C}$, respectively. The annealed samples on the fixed glass slides were mechanically sheared by stainless steel scrapers at a speed of $\sim 0.01 \mathrm{~m} / \mathrm{s}$. For polymeric samples, the sheared films were further annealed at $\sim 150{ }^{\circ} \mathrm{C}$ for several hours. Finally, the sheared films were peeled off by razor blades and exposed to X-ray beam through different directions. The thicknesses of the oriented films maintain at $\sim 3 \mathrm{~mm}$. 
Density Measurement. Experimental densities of polymers were detected by the floating method. Mixed solutions of methanol/saturated $\mathrm{NaCl}$ aqueous solution with different volume-ratios were used as floating mediums. Samples $(\sim 15 \mathrm{mg})$ on glass slides were heated to high-temperatures at which they were dressed by stainless steel scrapers and then cooled to RT, affording regular-shaped solids. The densities of the solids were then determined according to the typical floating procedure.

\section{Synthesis and Molecular Characterization}

\subsection{Synthesis}

The synthetic routes to phasmidic mesogens (MS- $n, n=8,12$ ), monomers (ME- $n$ and MNB- $n, n=8,12$ ), and polymers (PE- $n$ and PNB- $n, n=8,12)$ are given in Figure S1. Key intermediates $\mathbf{1 - n}(n=8,12), \mathbf{3}$, and $\mathbf{4}$ were synthesized according to the reported methods. ${ }^{1-3}$ Therification of $\mathbf{1 - n}(n=8,12)$ with $\mathbf{2}, \mathbf{3}$, and $\mathbf{4}$ in the presence of dicyclohexylcarbodiimide (DCC) and 4-dimethylaminopyridine (DMAP) produced MS- $n$, ME- $n$, and MNB- $n(n=8,12)$, respectively. Radical polymerization and ring-opening metathesis polymerization (ROMP) of ME- $n$ and MNB- $n$ result in PE- $n$ and PNB- $n(n=8,12)$, respectively. 


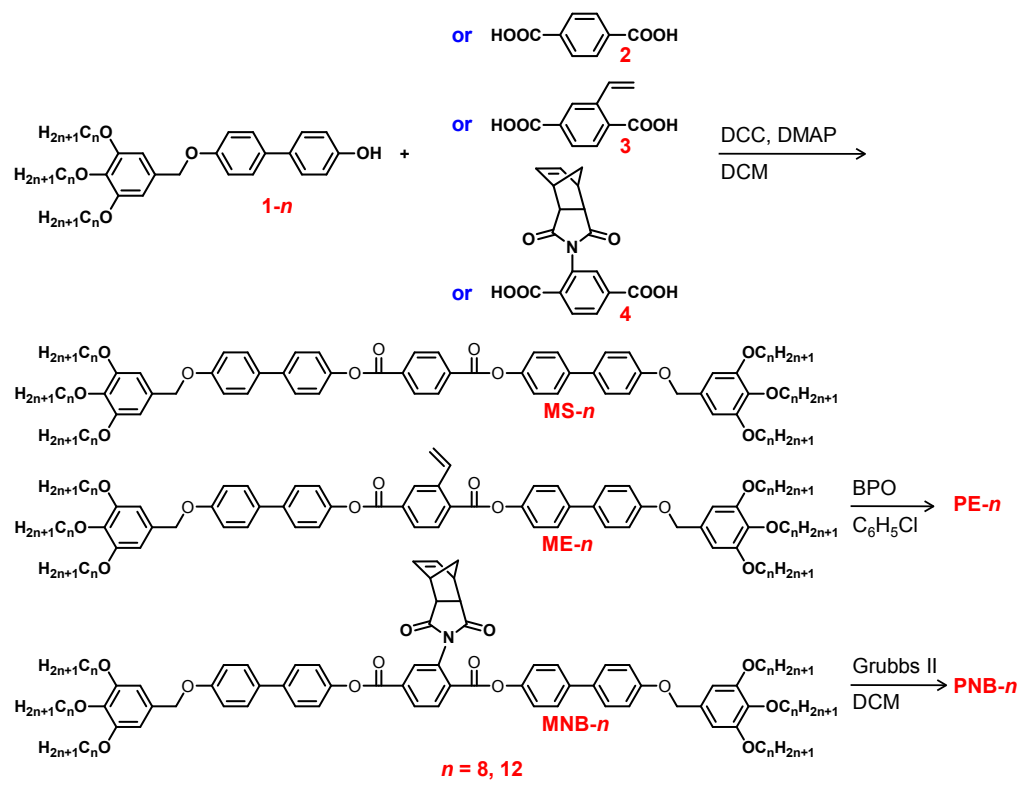

Figure S1. Synthetic routes to phasmidic molecules (MS- $n, n=8,12$ ), monomers (ME- $n$ and MNB- $n, n=8,12$ ), and polymers (PE- $n$ and PNB- $n, n=8,12$ ).

A typical procedure for therification. As an example, the synthesis of ME-12 is described in detail below. 1-12 (2.00 g, $2.41 \mathrm{mmol}), 3(0.23 \mathrm{~g}, 1.20 \mathrm{mmol})$, DCC $(0.54 \mathrm{~g}, 2.64 \mathrm{mmol})$, and DMAP $(0.032 \mathrm{~g}, 0.26 \mathrm{mmol})$ were dissolved in dry DCM $(10 \mathrm{ml})$ at room temperature $(\mathrm{RT})$. The reaction mixture was further stirred at RT for $48 \mathrm{~h}$. After filtration, the solvent was removed under reduced pressure. The crude product was purified by silica gel chlomatography with DCM as the eluent and then recrystallized with ethanol to give ME-12 as a white crystalline solid (1.64 g, 75\%).

A typical procedure for radical polymerization. As an example, the polymerization of ME-12 is described in detail below. Monomer ME-12 (250 mg, $0.14 \mathrm{mmol})$, BPO (0.68 mg, $0.0028 \mathrm{mmol})$ in $80 \mu \mathrm{L}$ of $\mathrm{C}_{6} \mathrm{H}_{5} \mathrm{Cl}$, and $\mathrm{C}_{6} \mathrm{H}_{5} \mathrm{Cl}(0.5 \mathrm{ml})$ were successfully introduced a polymerization tube. After three freeze-pump-thaw cycles, the tube was sealed off under vacuum. The solution was further stirred at 90 
${ }^{\circ} \mathrm{C}$ for $12 \mathrm{~h}$. The tube was then opened and the reaction mixture was diluted with $2 \mathrm{ml}$ of tetrahydrofuran (THF). The diluted solution was dropped into $50 \mathrm{ml}$ of THF-methanol $(1: 2.5, v / v)$ mixture. The precipitate was collected by filtration. The dissolution-precipitation process was repeated three times. After drying under vacuum, $180 \mathrm{mg}$ of polymer was obtained in $72 \%$ yield.

A typical procedure for ROMP polymerization. As an example, the polymerization of MNB-12 is described in detail below. Monomer MNB-12 (250 mg, $0.128 \mathrm{mmol})$ and Grubbs II (4.61 $\mathrm{mg}, 0.0016 \mathrm{mmol})$ were loaded in a dry Schlenk tube with a magnetic stirring bar. After three pump-purge cycles with high purity nitrogen, DCM (1 ml) was injected to the mixture under vigorous stirring. After the reaction mixture was stirred at $30{ }^{\circ} \mathrm{C}$ for $2 \mathrm{~h}$, a few drops of vinyl ethyl ether were added to the reaction mixture using a syringe. After an additional $1 \mathrm{~h}$, the polymerization was stopped. The viscous liquid was diluted with $2 \mathrm{~mL}$ THF and passed through a short alumina column to remove the catalyst with DCM as the eluent. The collected solution was concentrated to about $2 \mathrm{ml}$, and then dropped into $50 \mathrm{ml}$ of THF-methanol $(1: 2.5, v / v)$ mixture. The precipitate was collected by filtration. The dissolution-precipitation process was repeated three times. After drying under vacuum, $175 \mathrm{mg}$ of polymer was obtained in $70 \%$ yield.

\subsection{Molecular Characterization}

NMR spectra of key intermediates (1-n, 3, and 4), phasmidic mesogens (MS- $n, n=$ 8, 12), monomers (ME- $n$ and MBN- $n, n=8,12$ ), and polymers (PE- $n$ and PNB- $n, n=$ 
$8,12)$ are given below. And the results of GPC of PE- $n$ and PNB- $n(n=8,12)$ are also provided below.

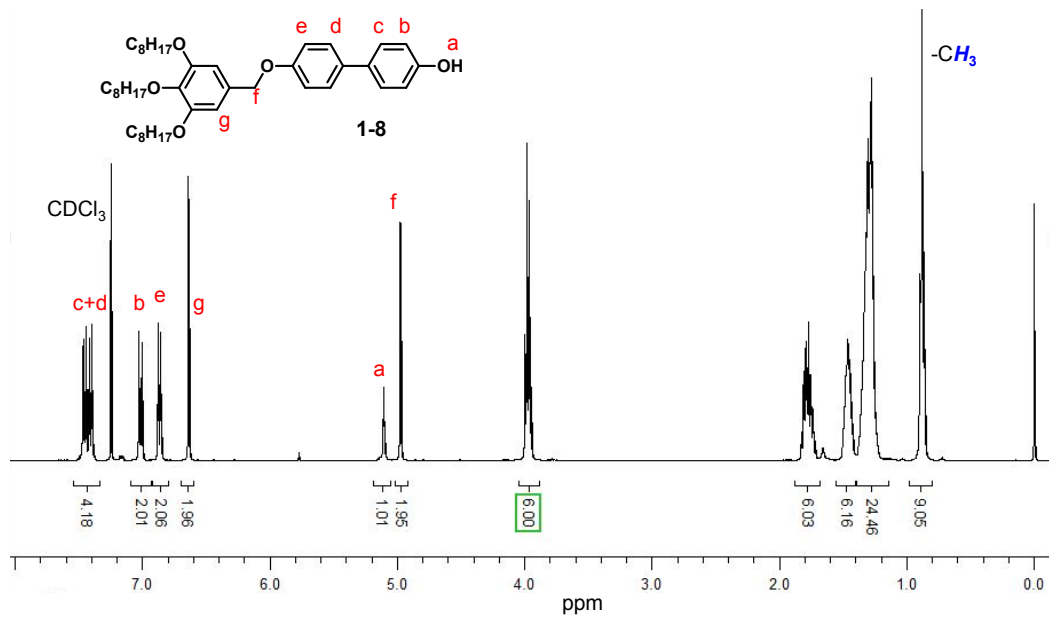

Figure S2. ${ }^{1} \mathrm{H}$ NMR spectrum of $\mathbf{1 - 8}$ in $\mathrm{CDCl}_{3}$.

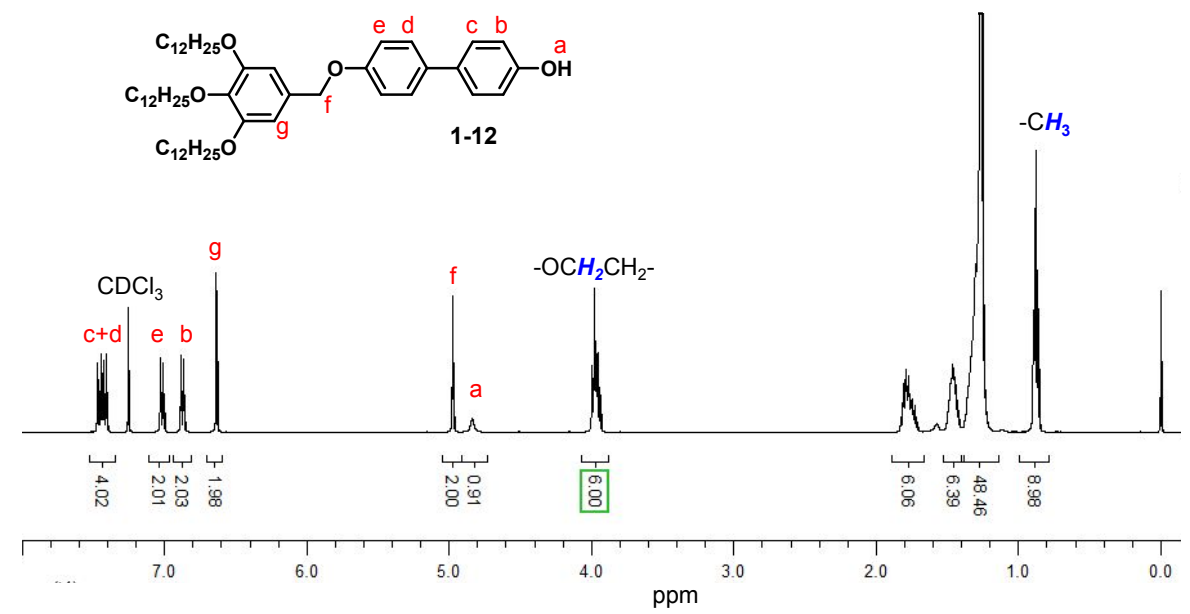

Figure S3. ${ }^{1} \mathrm{H}$ NMR spectrum of 1-12 in $\mathrm{CDCl}_{3}$. 


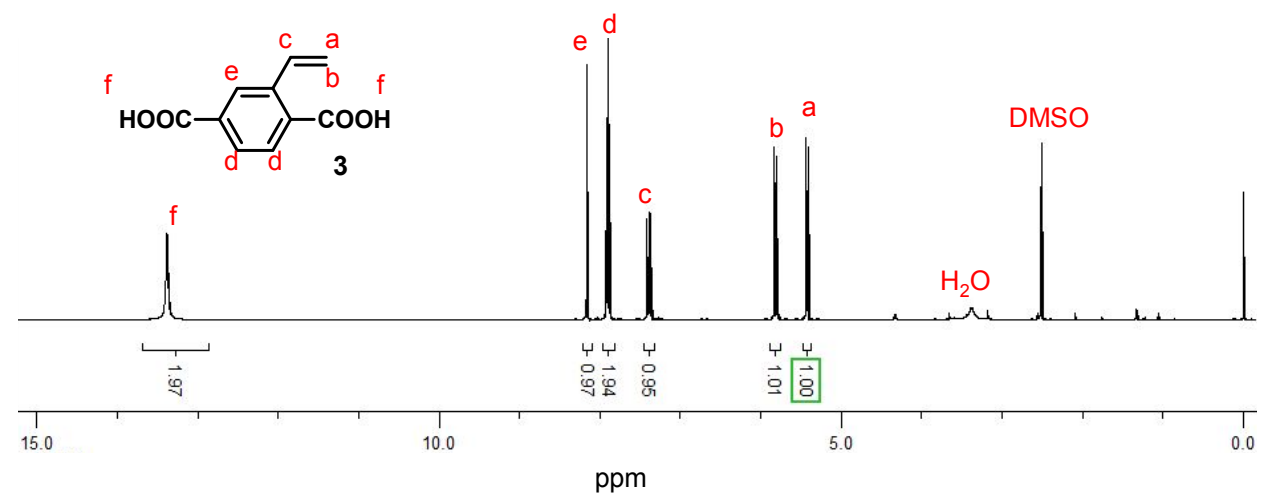

Figure $\mathbf{S 4} .{ }^{1} \mathrm{H}$ NMR spectrum of 3 in DMSO.

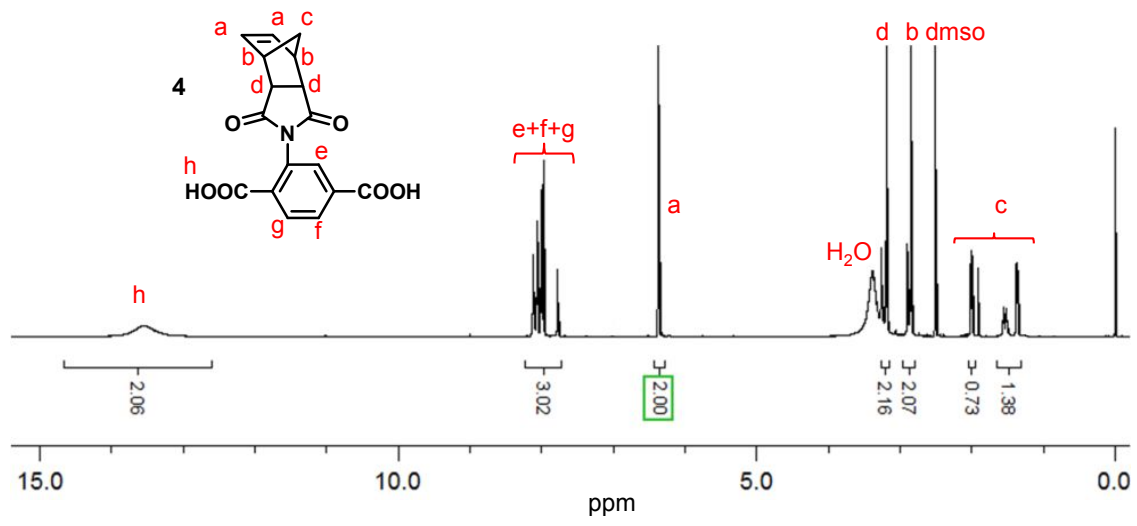

Figure S5. ${ }^{1} \mathrm{H}$ NMR spectrum of 4 in DMSO.

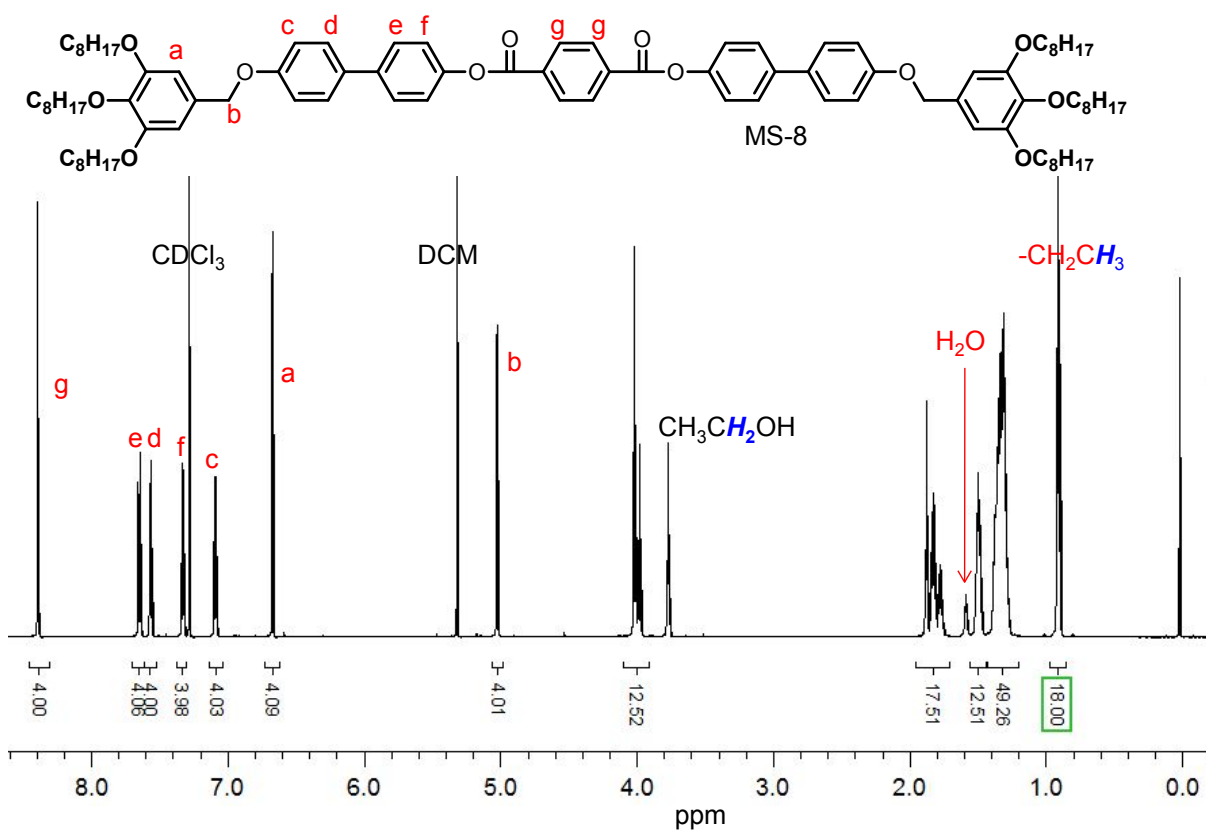

Figure S6. ${ }^{1} \mathrm{H}$ NMR spectrum of MS-8 in $\mathrm{CDCl}_{3}$. 


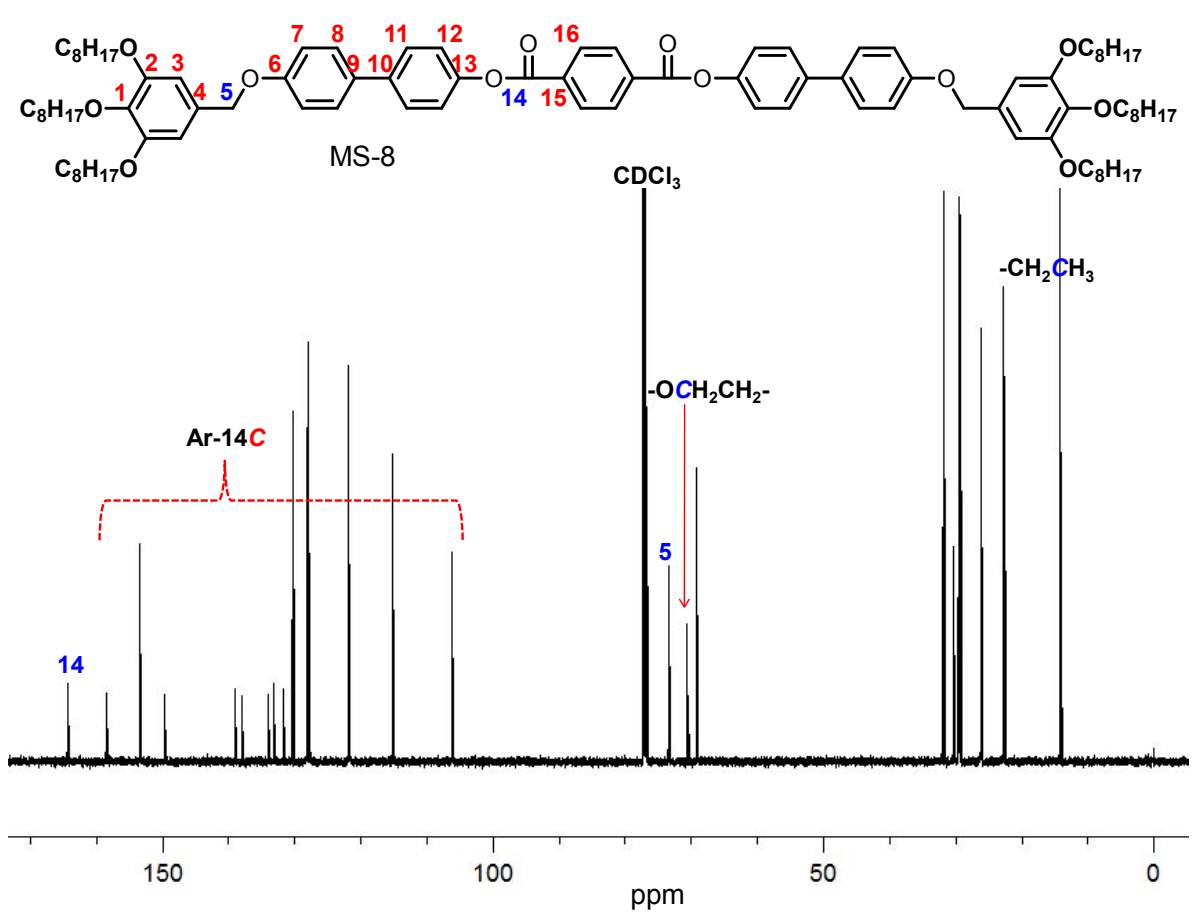

Figure S7. ${ }^{13} \mathrm{C}$ NMR spectrum of $\mathrm{MS}-8$ in $\mathrm{CDCl}_{3}$.

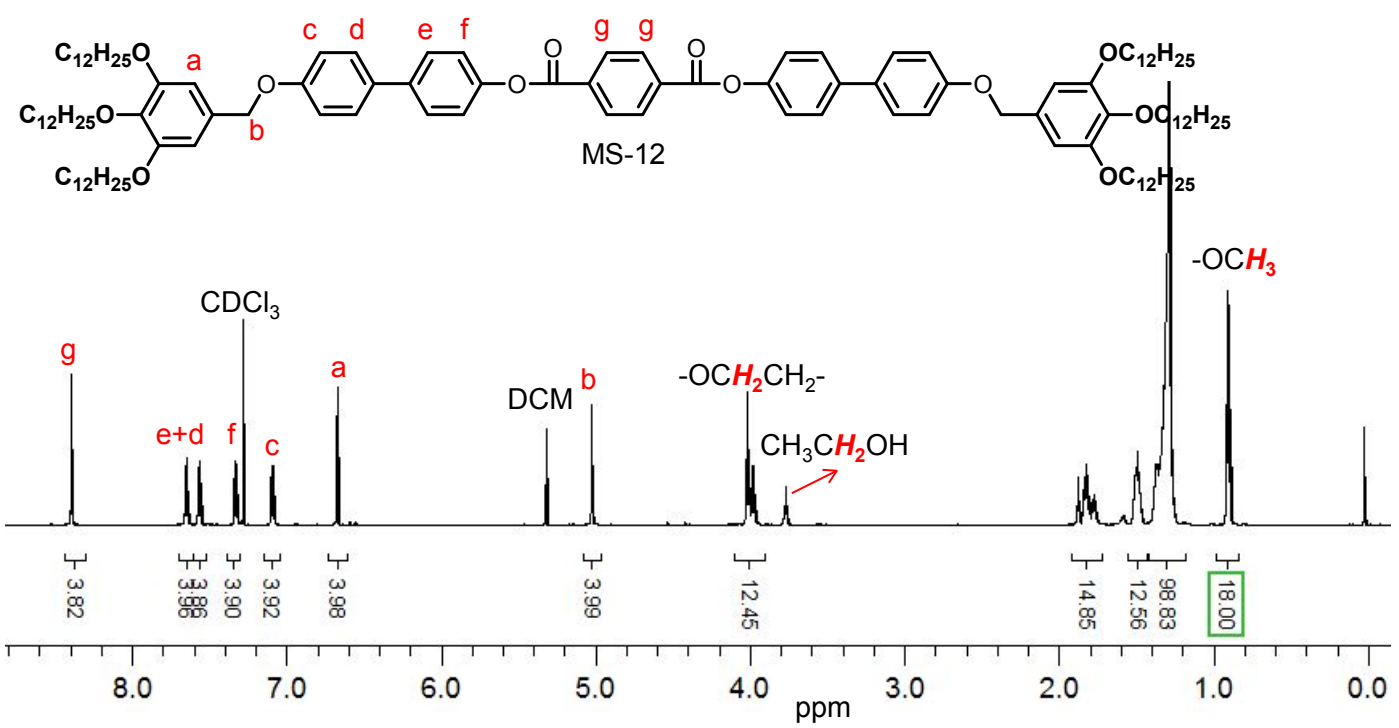

Figure S8. ${ }^{1} \mathrm{H}$ NMR spectrum of MS-12 in $\mathrm{CDCl}_{3}$. 

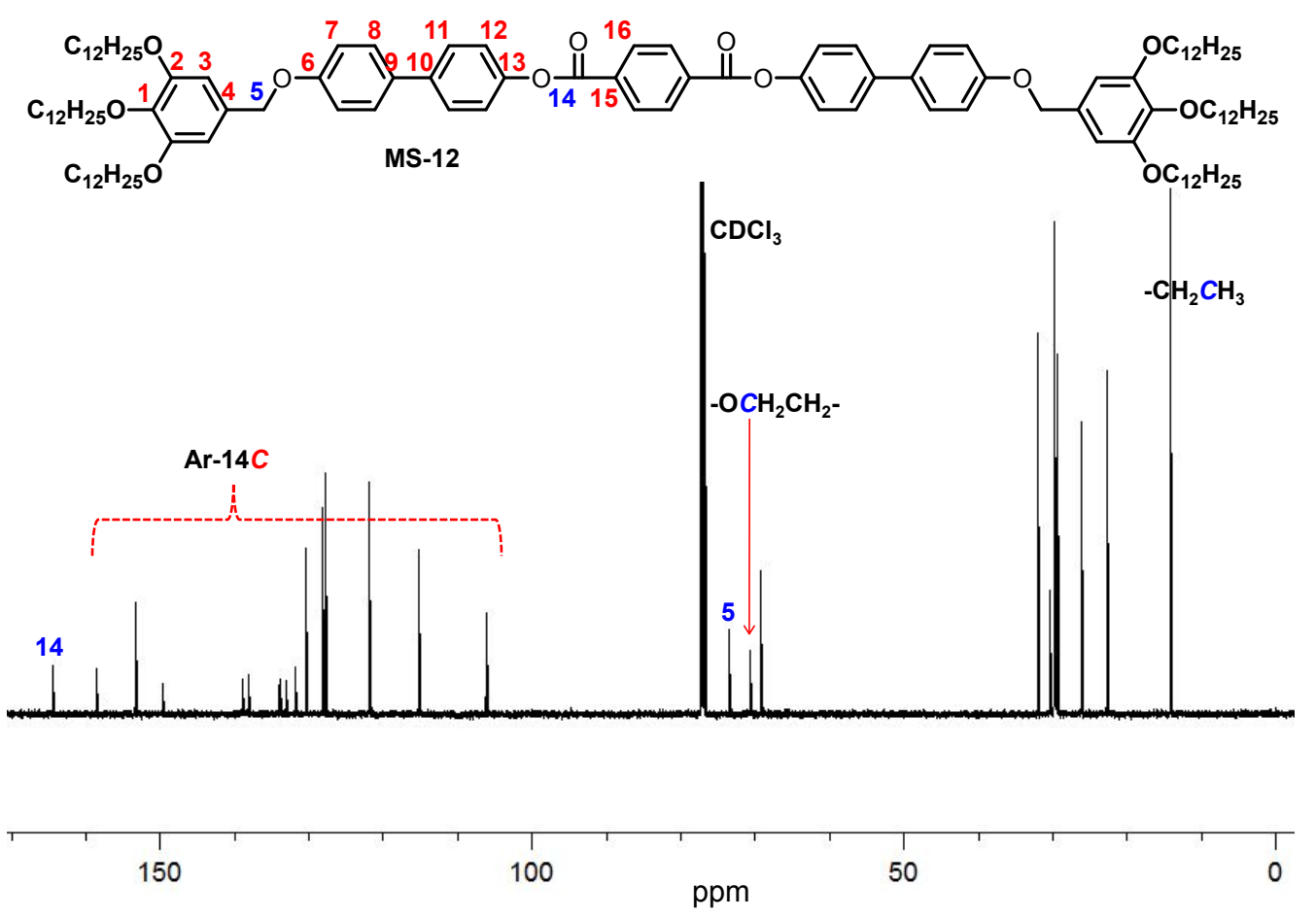

Figure S9. ${ }^{13} \mathrm{C}$ NMR spectrum of MS-12 in $\mathrm{CDCl}_{3}$.

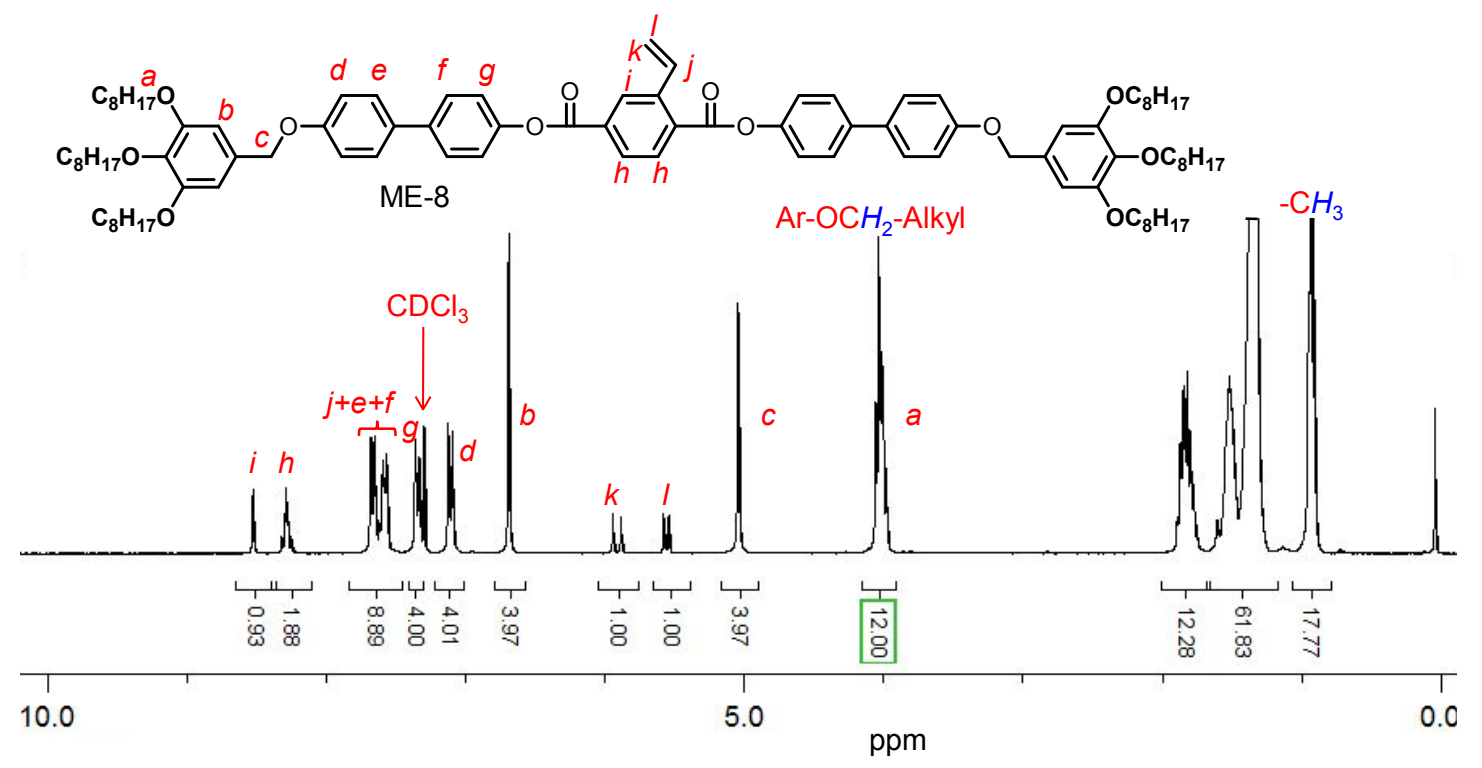

Figure S10. ${ }^{1} \mathrm{H}$ NMR spectrum of ME-8 in $\mathrm{CDCl}_{3}$. 


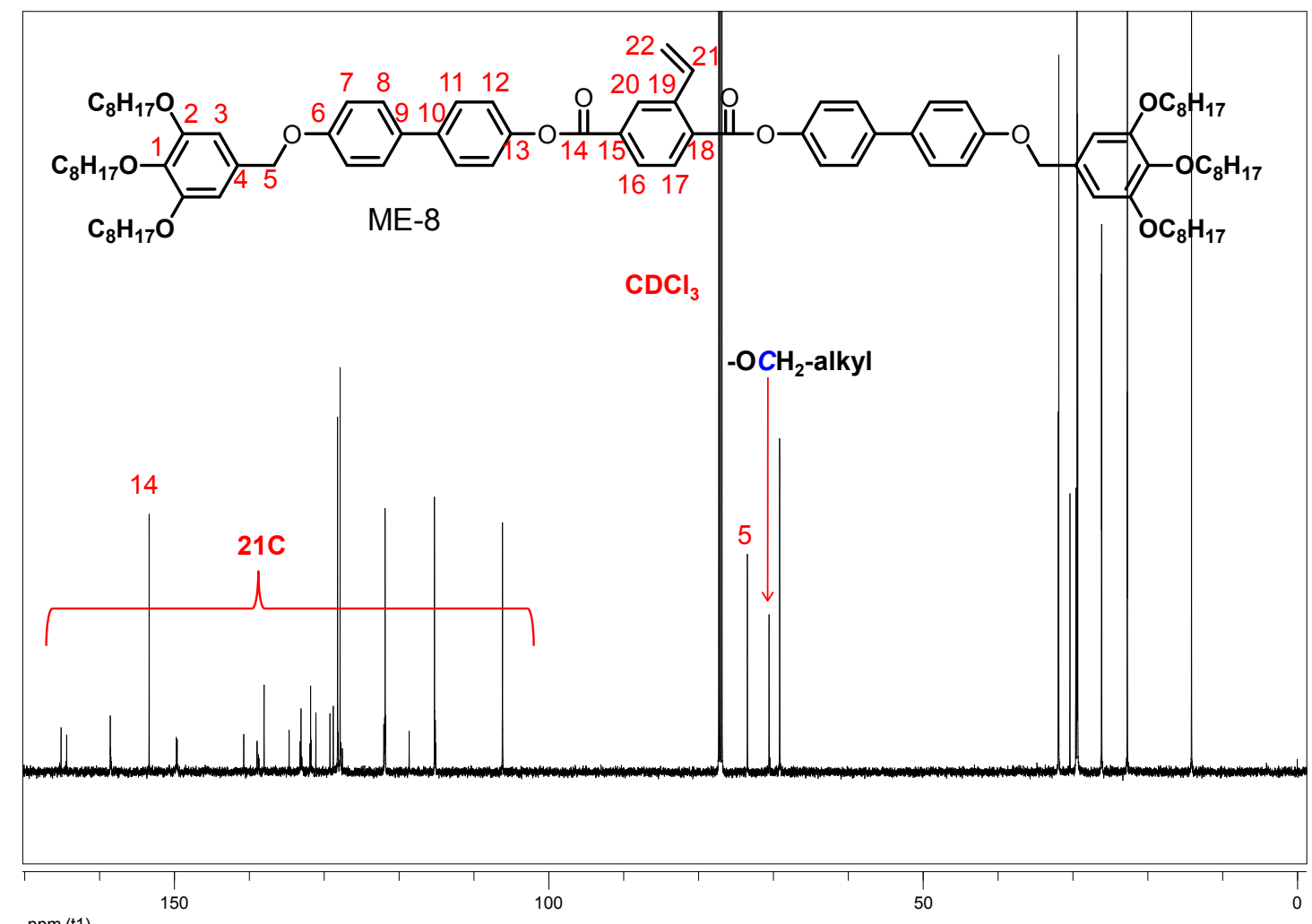

Figure S11. ${ }^{13} \mathrm{C}$ NMR spectrum of $\mathrm{ME}-8$ in $\mathrm{CDCl}_{3}$.

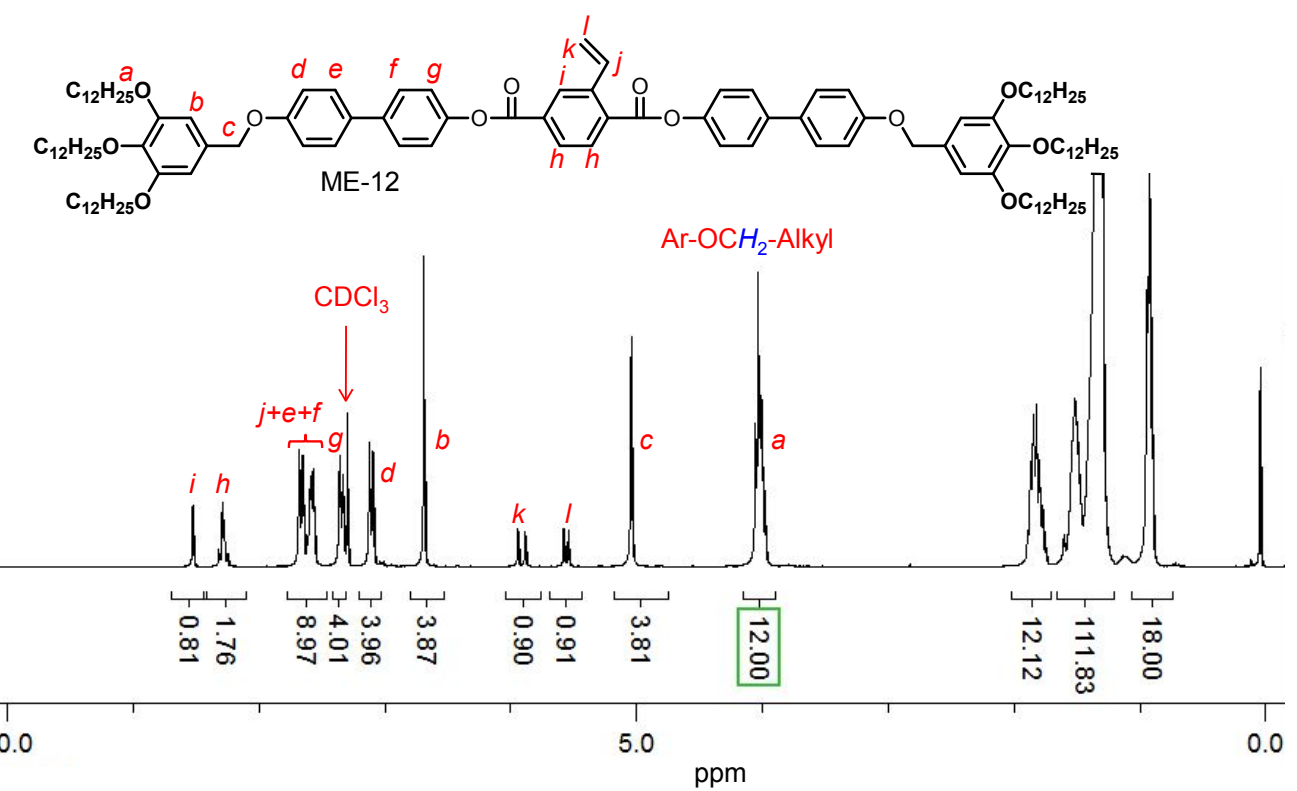

Figure S12. ${ }^{1} \mathrm{H}$ NMR spectrum of $\mathrm{ME}-12$ in $\mathrm{CDCl}_{3}$. 


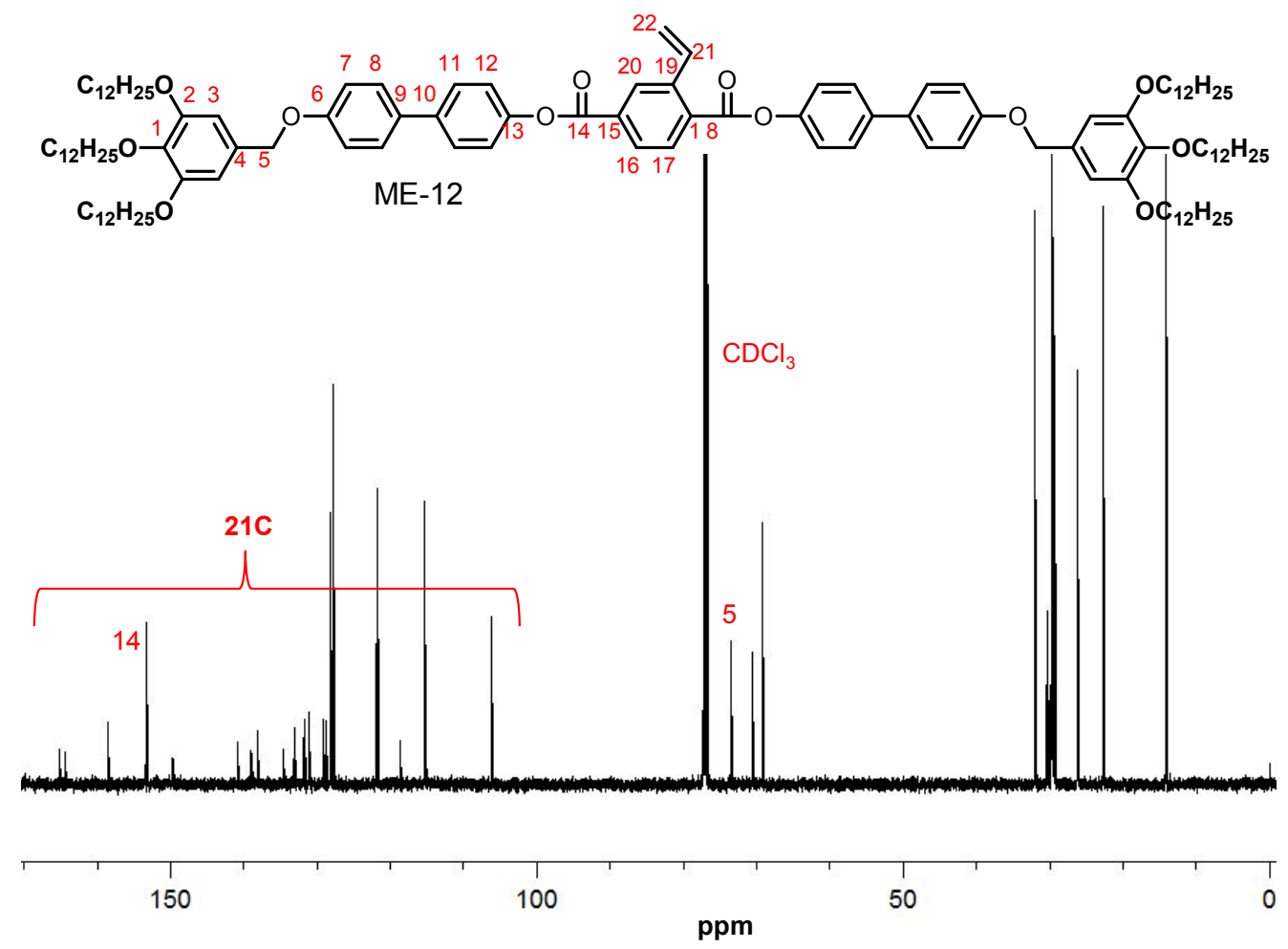

Figure S13. ${ }^{13} \mathrm{C}$ NMR spectrum of ME-12 in $\mathrm{CDCl}_{3}$.

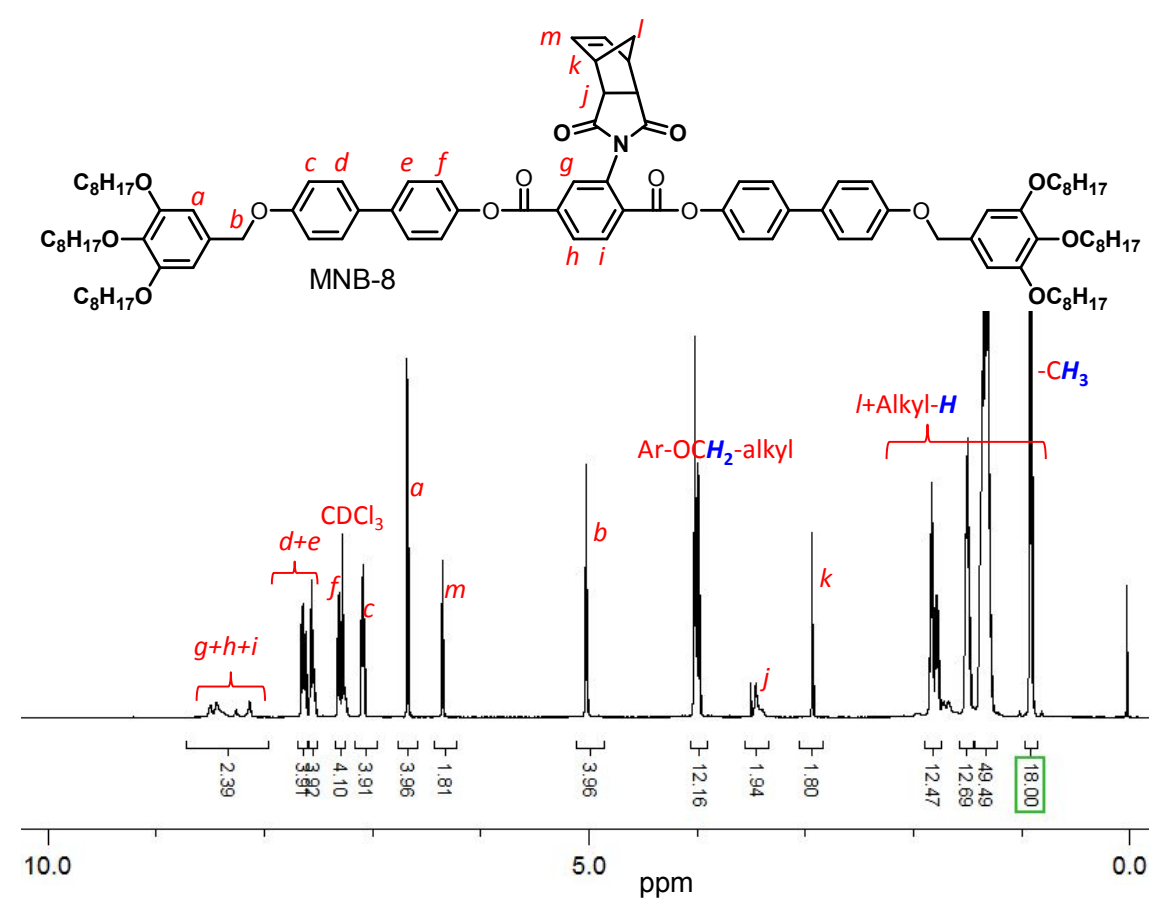

Figure S14. ${ }^{1} \mathrm{H}$ NMR spectrum of $\mathrm{MNB}-8$ in $\mathrm{CDCl}_{3}$. 


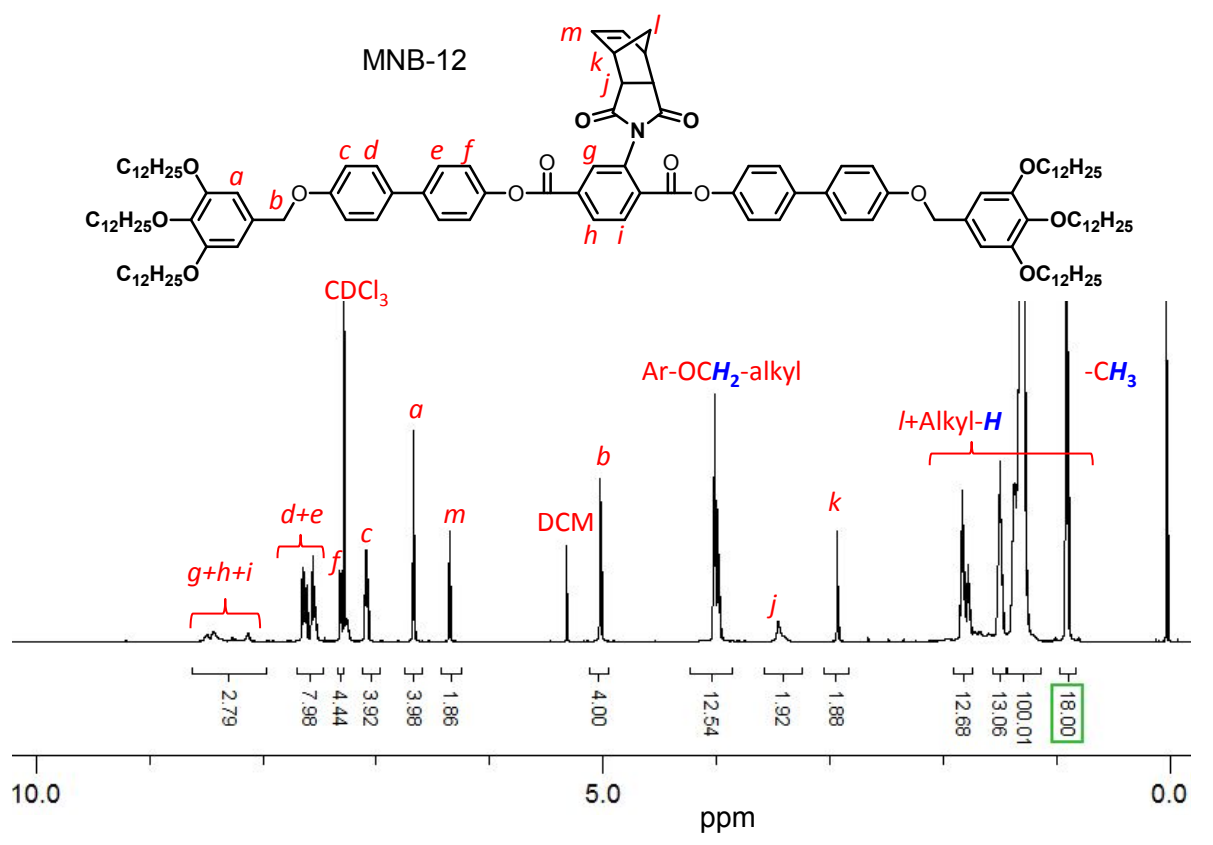

Figure S15. ${ }^{1} \mathrm{H}$ NMR spectrum of MNB-12 in $\mathrm{CDCl}_{3}$.

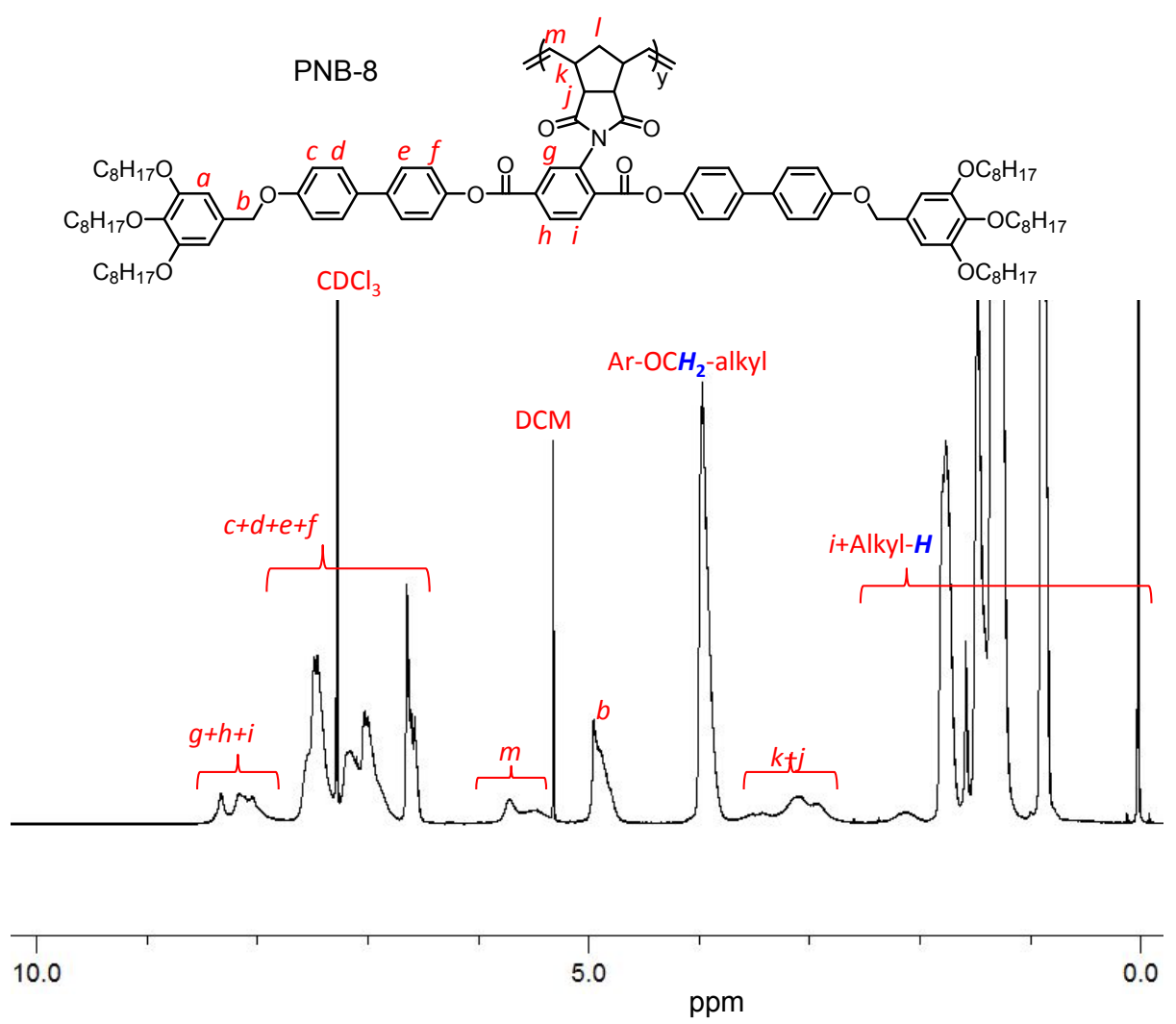

Figure S16. ${ }^{1} \mathrm{H}$ NMR spectrum of PNB-8 in $\mathrm{CDCl}_{3}$. 

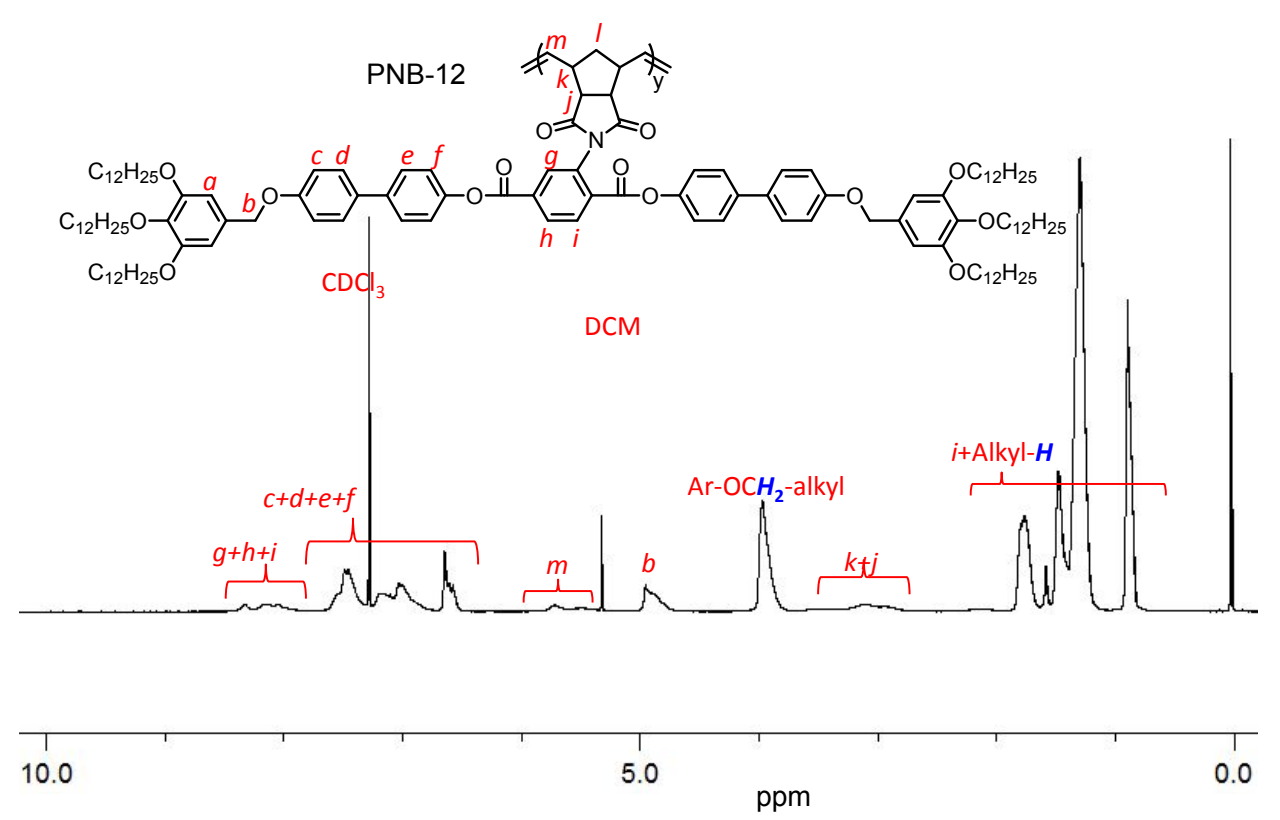

Figure S17. ${ }^{1} \mathrm{H}$ NMR spectrum of PNB-12 in $\mathrm{CDCl}_{3}$.

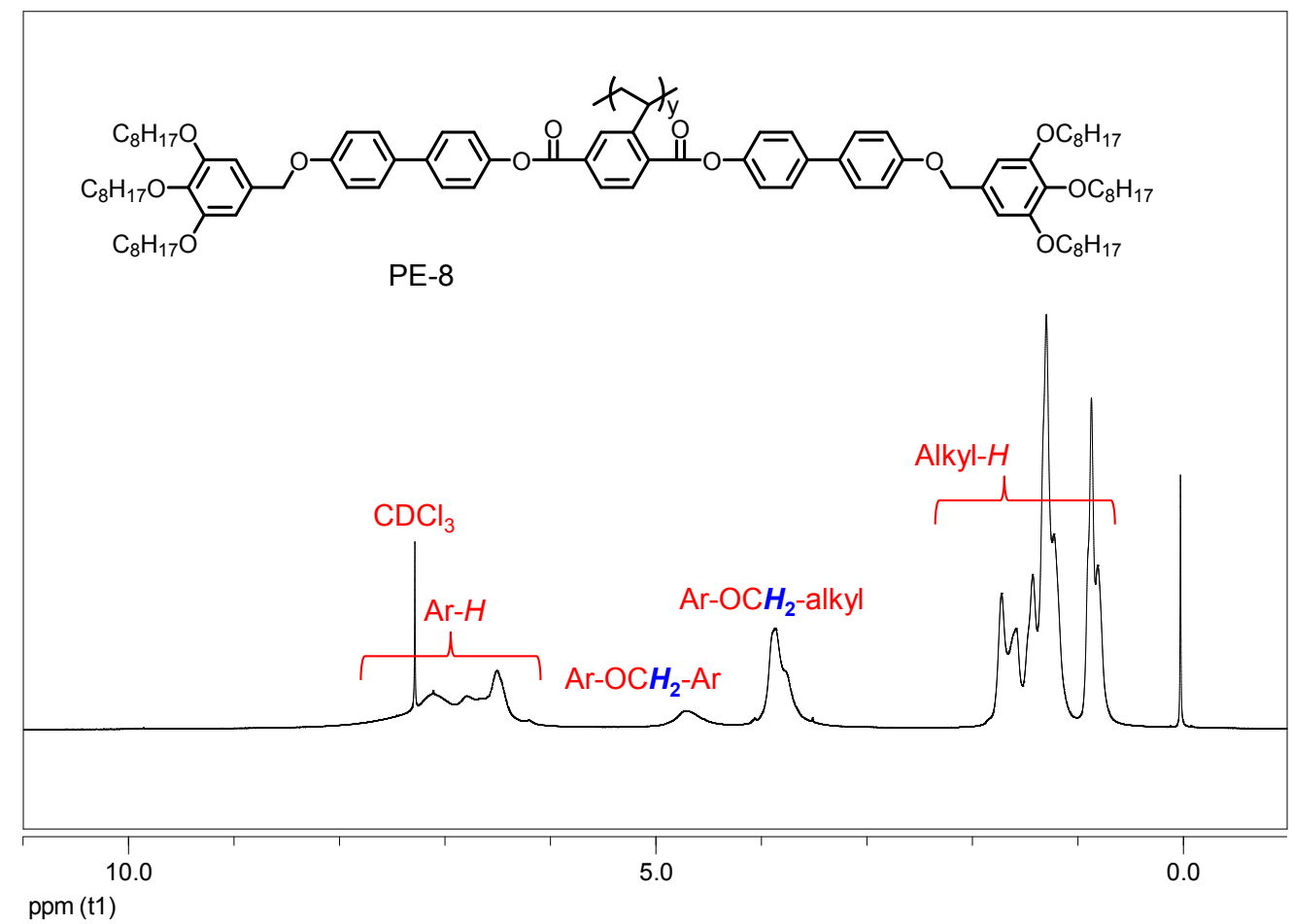

Figure S18. ${ }^{1} \mathrm{H}$ NMR spectrum of $\mathrm{PE}-8$ in $\mathrm{CDCl}_{3}$. 


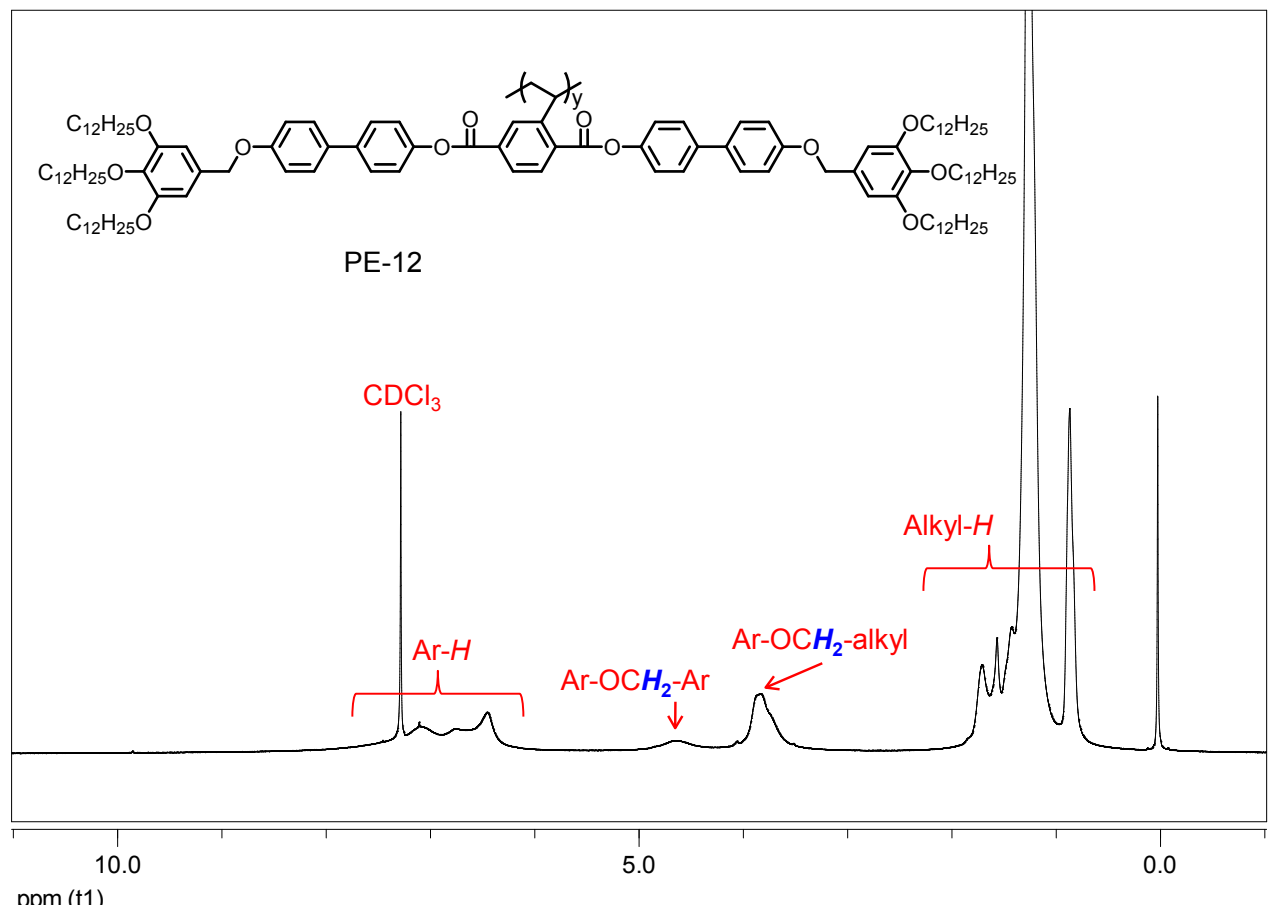

Figure S19. ${ }^{1} \mathrm{H}$ NMR spectrum of $\mathrm{PE}-12$ in $\mathrm{CDCl}_{3}$.

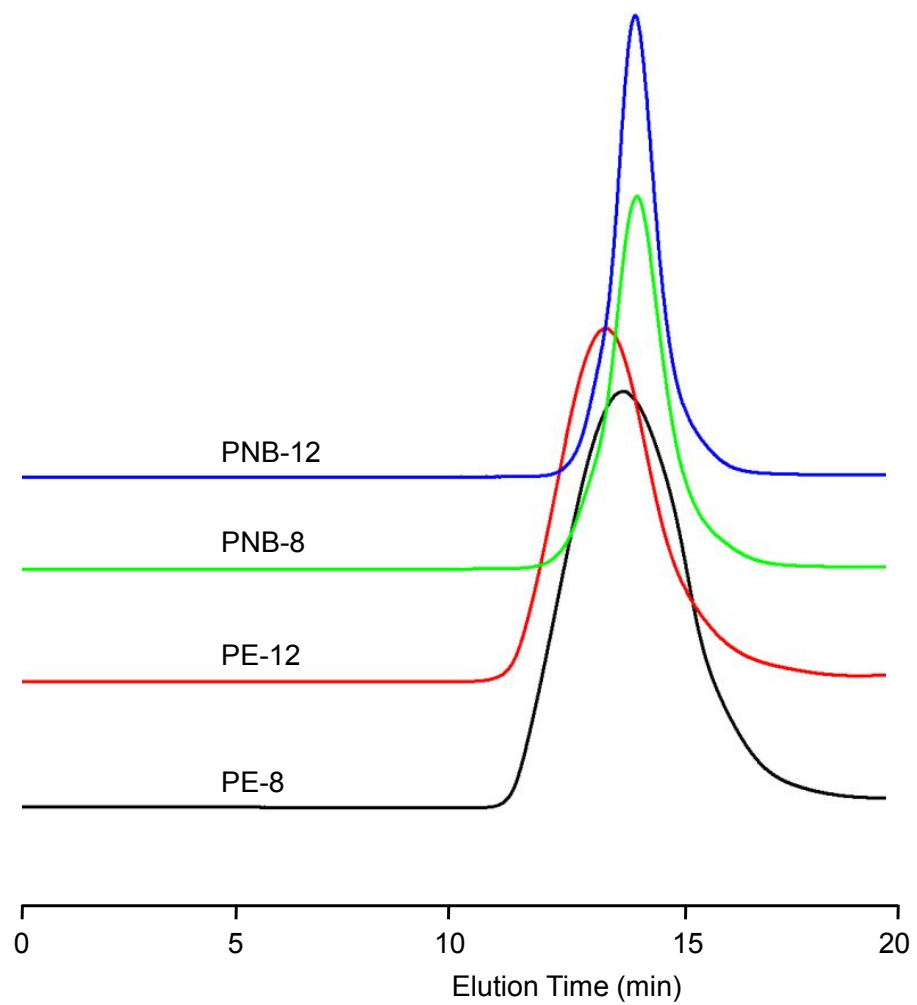

Figure S20. GPC traces of PE- $n$ and PNB- $n(n=8,12)$ 
Table S1. Number-average molecular weights $\left(M_{\mathrm{n}}\right)$ and polydispersity indexes (PDI) of PE- $n$ and PNB- $n(n=8,12)$.

\begin{tabular}{lcccc}
\hline & PE-8 & PE-12 & PNB-8 & PNB-12 \\
\hline$M_{\mathrm{n}}\left(\times 10^{5} \mathrm{~g} / \mathrm{mol}\right)$ & 1.68 & 1.98 & 1.28 & 1.25 \\
PDI & 1.75 & 1.60 & 1.12 & 1.10 \\
\hline
\end{tabular}

\section{Phase Structure Characterization}

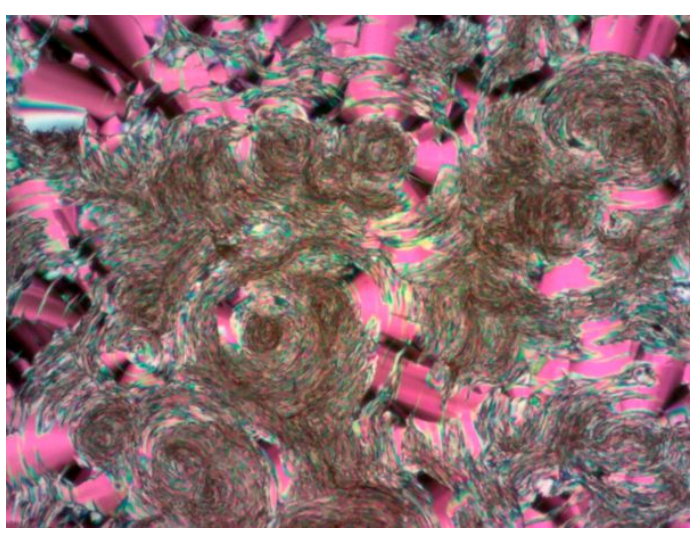

a

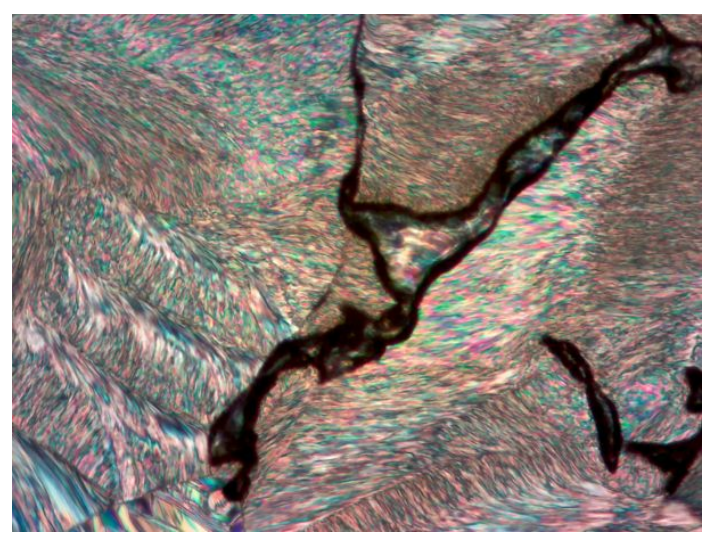

b

Figure S21. POM images of (a) MS- 8 taken at $70{ }^{\circ} \mathrm{C}$ and (b) MS-12 taken at $75{ }^{\circ} \mathrm{C}$ on cooling from their isotropic states. 

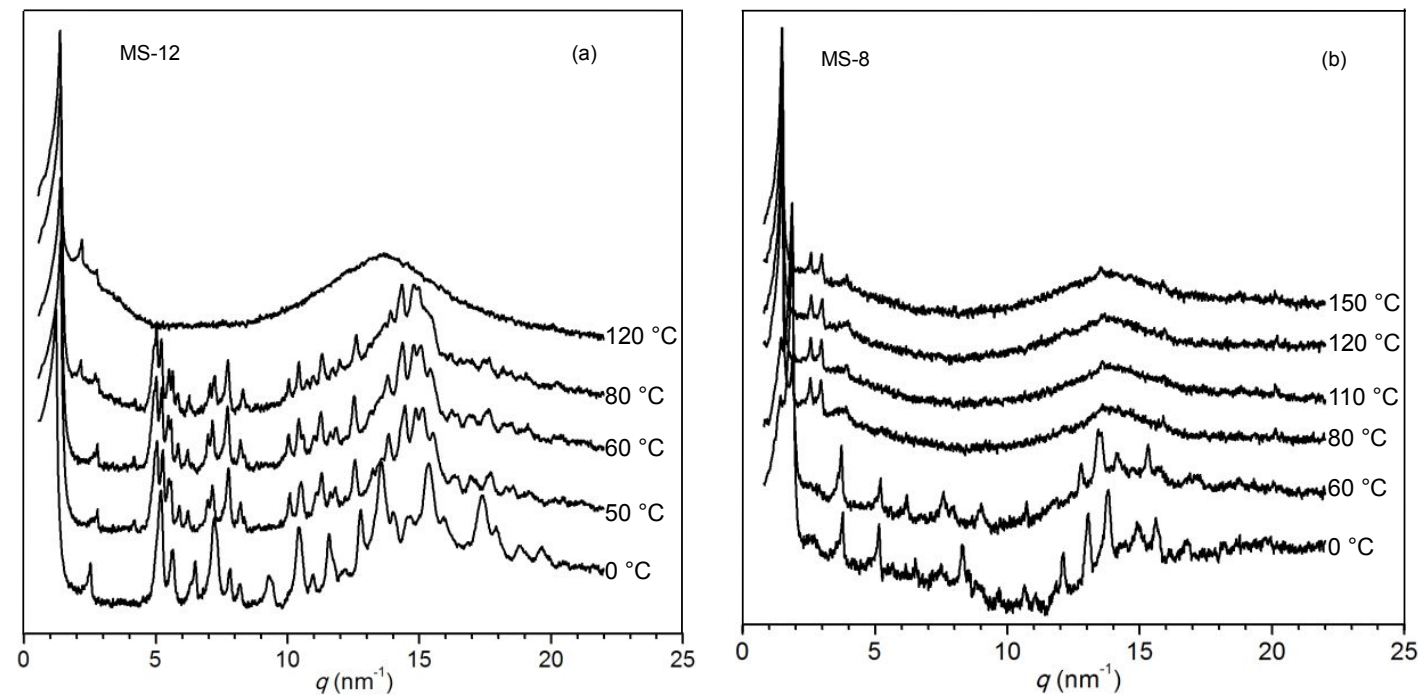

Figure S22. 1D XRD profiles of (a) MS-12 and (b) MS-8 collected upon cooling.
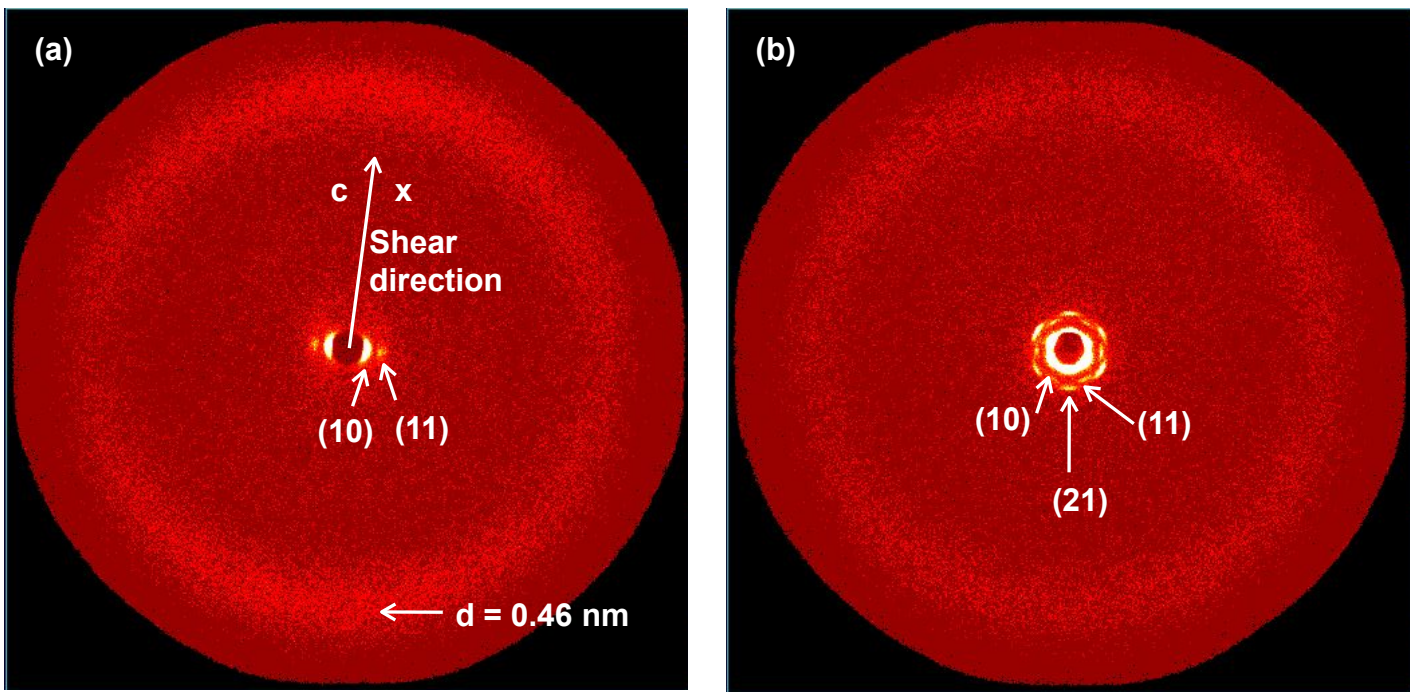

Figure S23. 2D XRD patterns collected at $90{ }^{\circ} \mathrm{C}$ of an oriented MS-12 film prepared by mechanical shearing, with X-ray beam perpendicular (a) and parallel (b) to the shear direction $x$. 


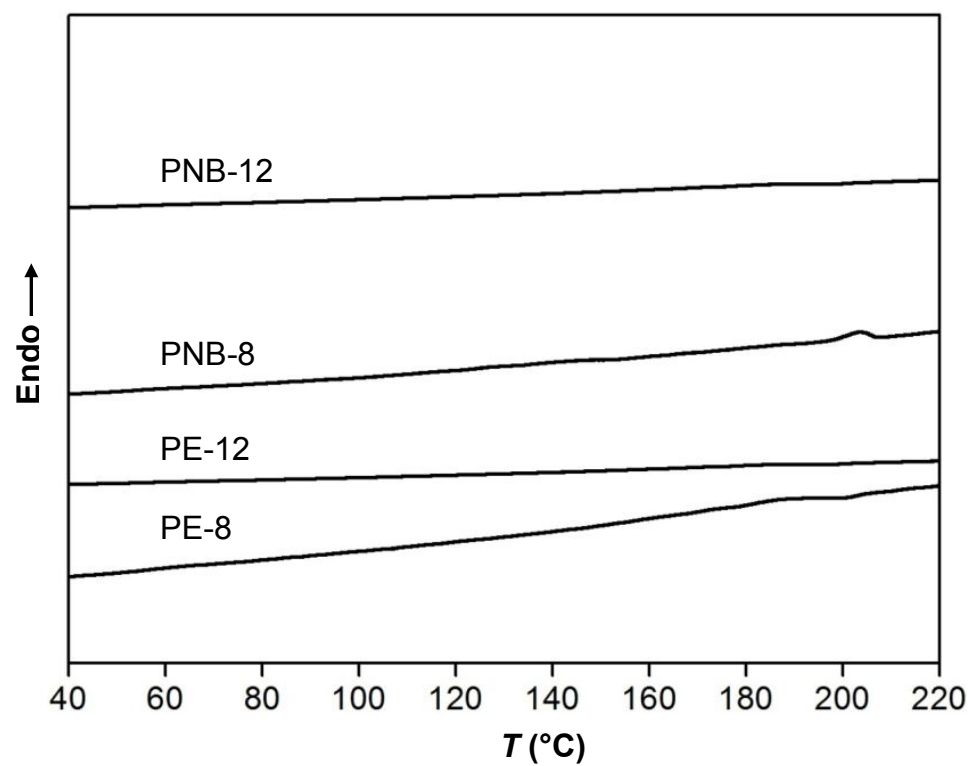

Figure S24. DSC traces of PE- $n$ and PNB- $n(\mathrm{n}=8,12)$ recorded on the second heating at $10{ }^{\circ} \mathrm{C} / \mathrm{min}$.

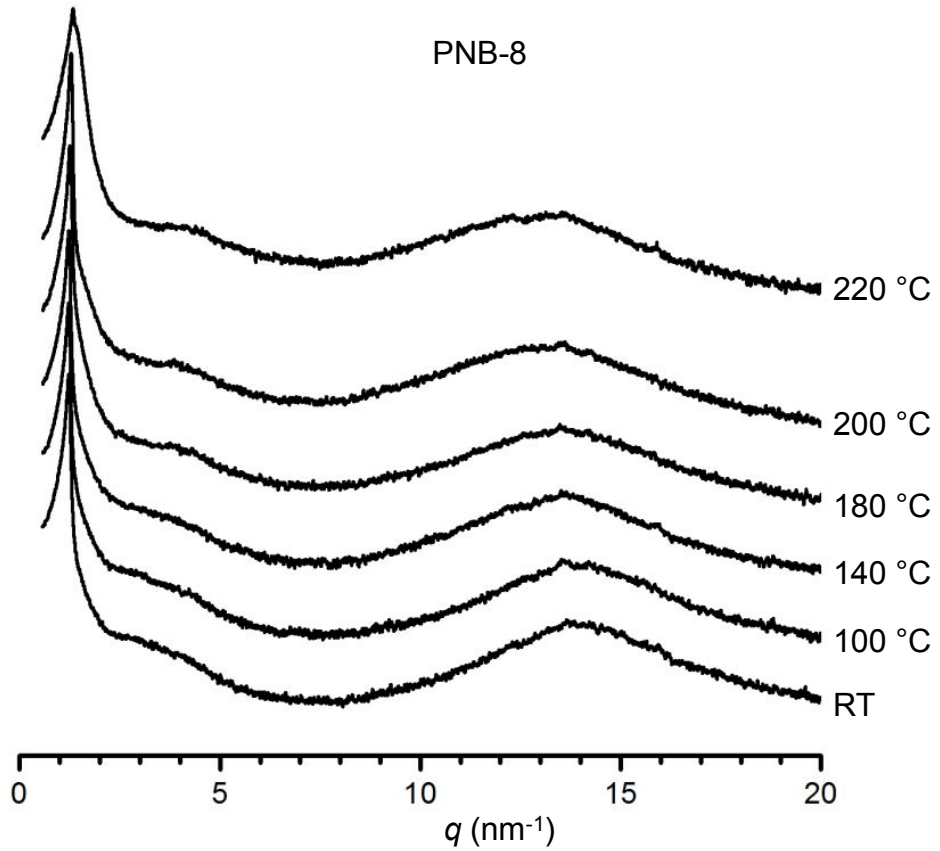

Figure S25. 1D XRD profiles of PNB-8 collected upon heating. 

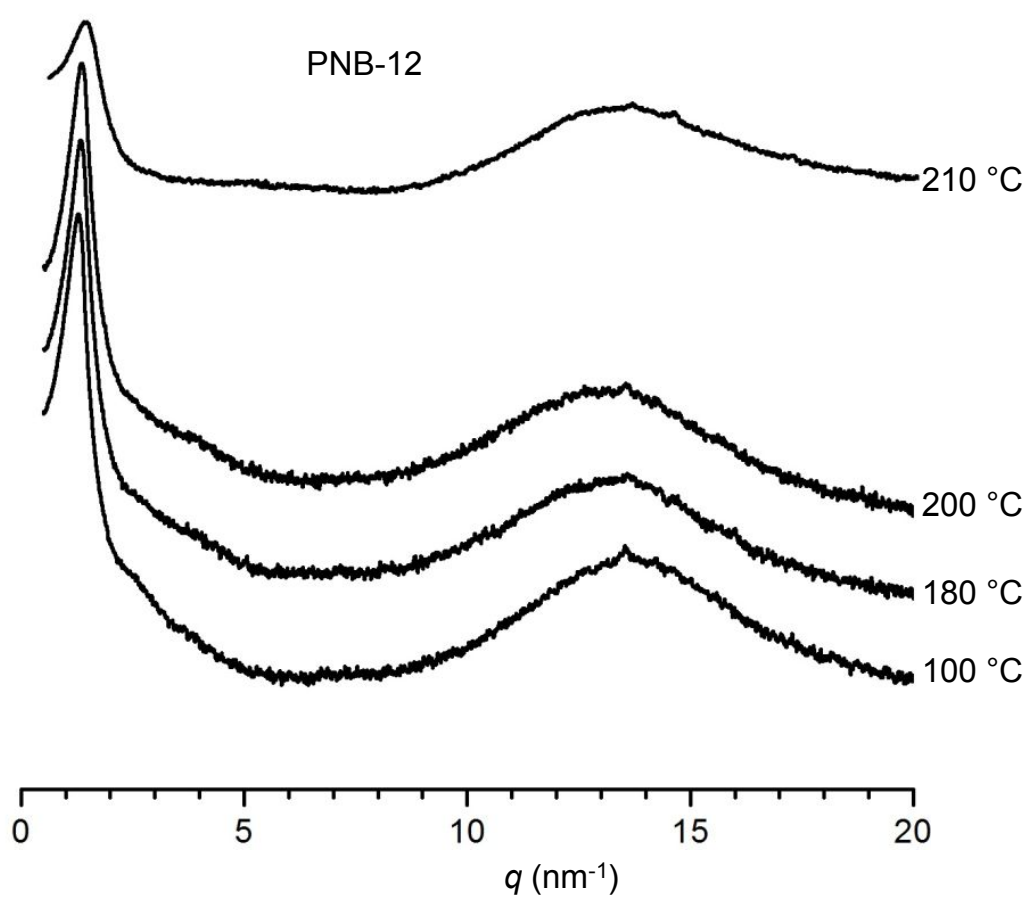

Figure S26. 1D XRD profiles of PNB-12 collected upon heating.

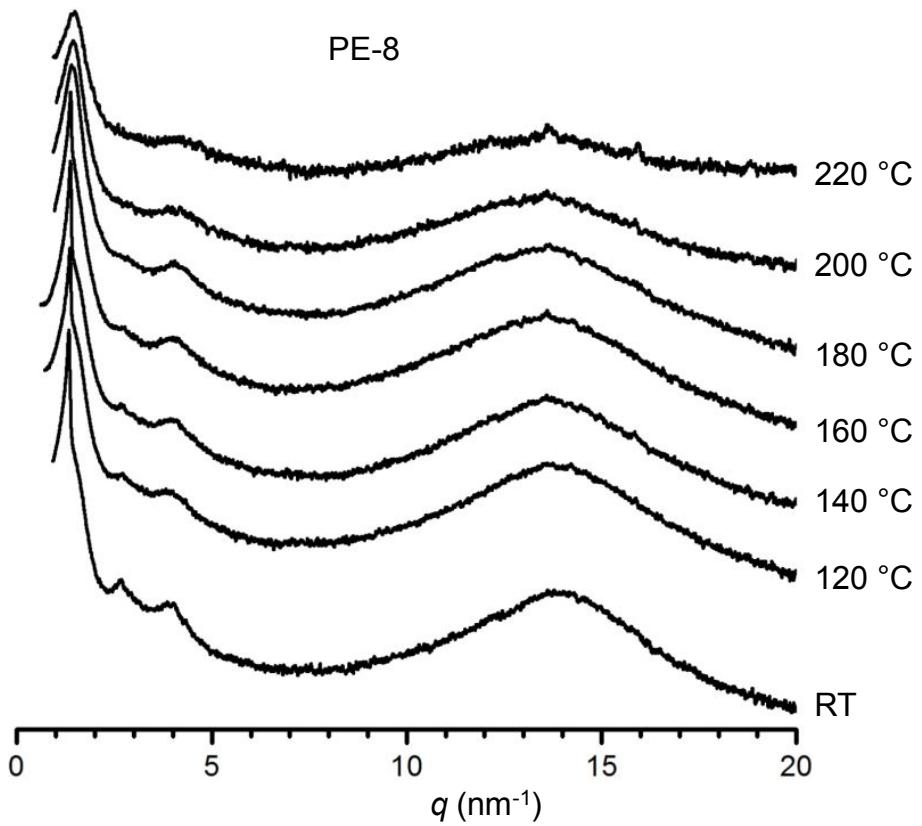

Figure S27. 1D XRD profiles of PE-8 collected upon heating. 


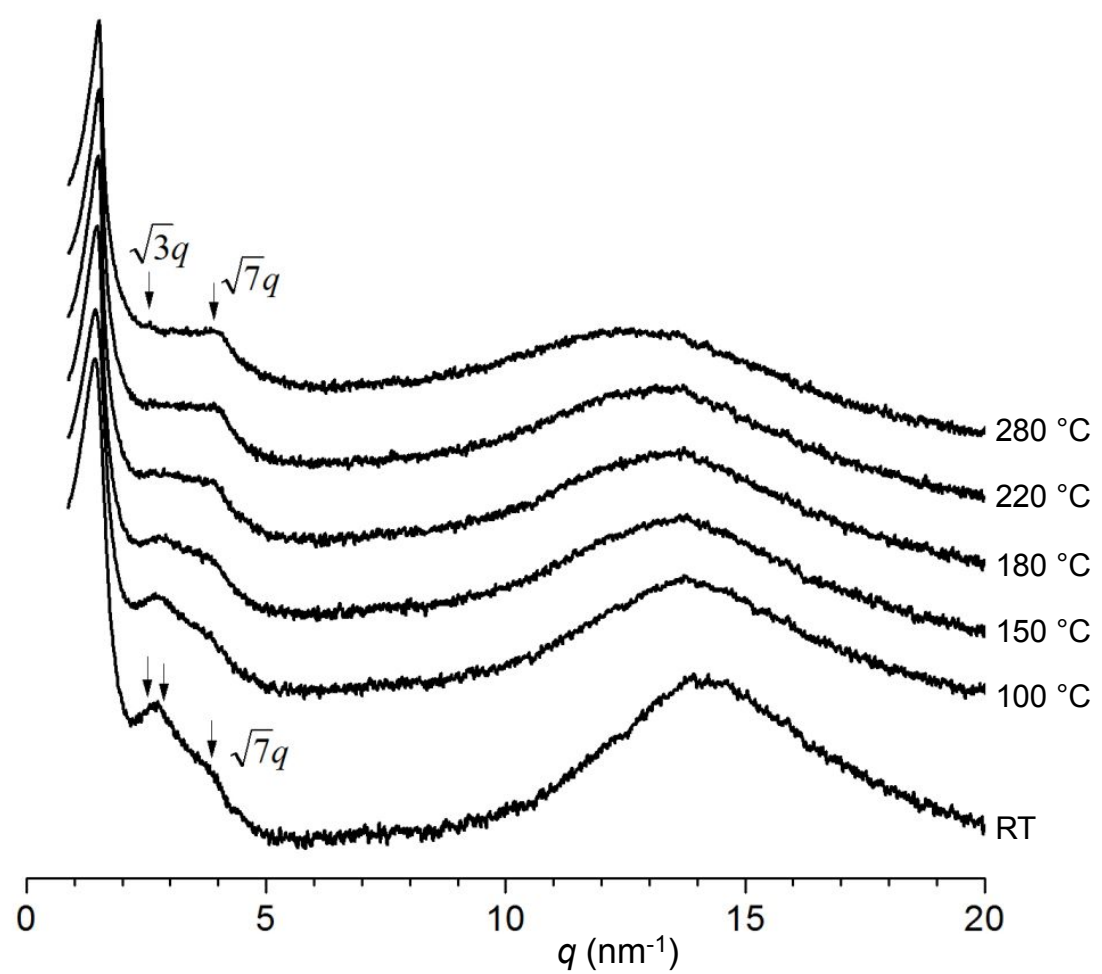

Figure S28. 1D XRD profiles of PE-12 collected upon heating.

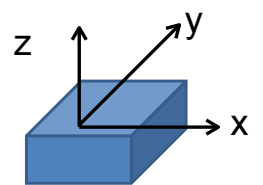

x: shear direction

z: shear gradient
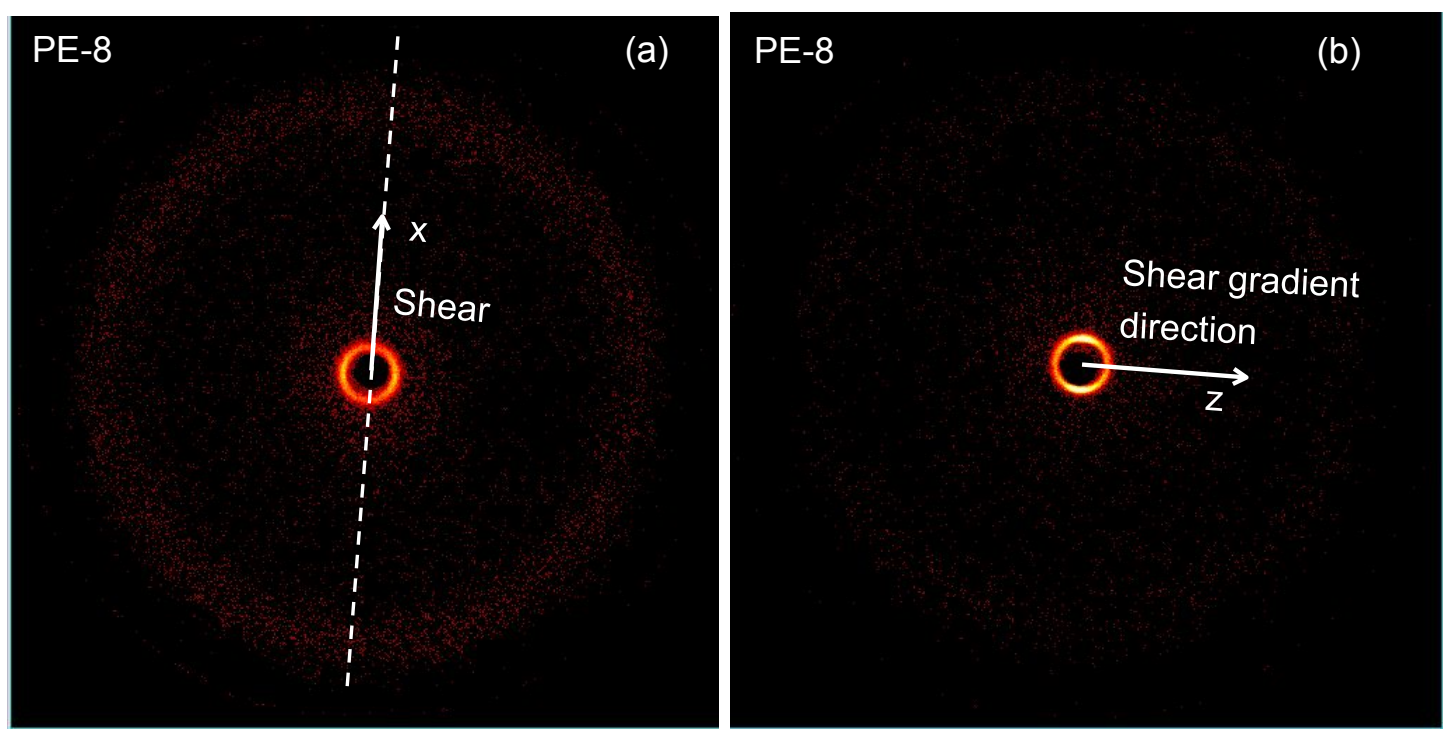

Figure S29. 2D XRD patterns of an oriented PE-8 film prepared by mechanical shearing with X-ray beam perpendicular (a) and parallel (b) to the shear direction $x$. 

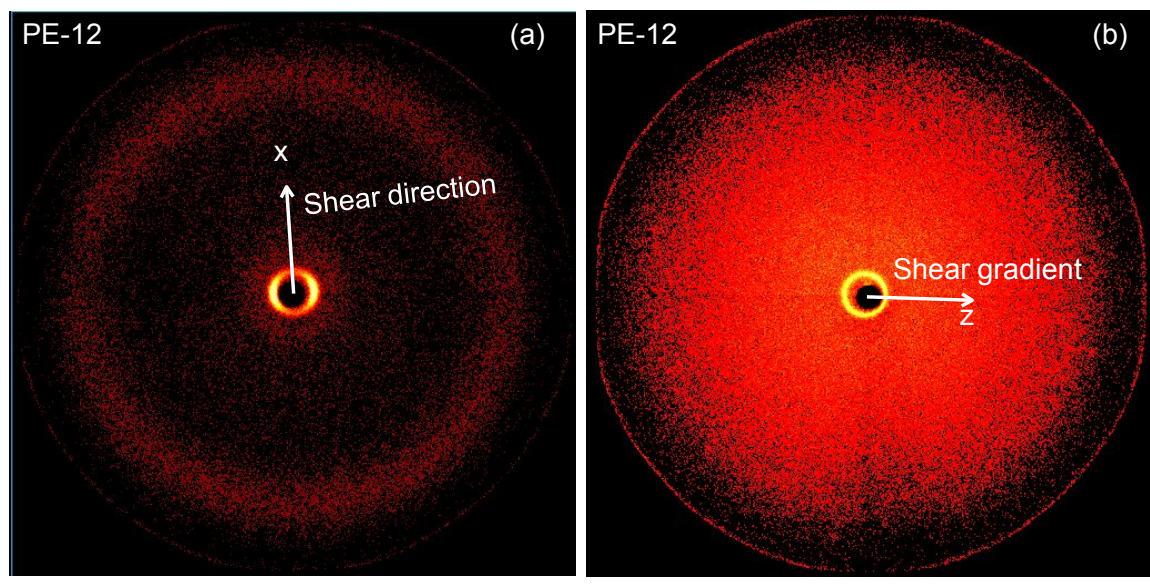

Figure S30. 2D XRD patterns of an oriented PE-12 film prepared by mechanical shearing with X-ray beam perpendicular (a) and parallel (b) to the shear direction $x$.

\section{References}

(1) Zheng, J.-F.; Liu, X.; Chen, X.-F.; Ren, X.-K.; Yang, S.; Chen, E.-Q. Hemiphasmidic Side-Chain Liquid Crystalline Polymer: From Smectic C Phase to Columnar Phase with a Bundle of Chains as Its Building Block. ACS Macro Lett. 2012, $1,641-645$.

(2) Zhou, Q.; Zhu, X.; Wen, Z. Liquid-crystalline side-chain polymers without flexible spacer. Macromolecules 1989, 22, 491-493.

(3) Zhu, Y.-F.; Zhang, Z.-Y.; Zhang, Q.-K.; Hou, P.-P.; Hao, D.-Z.; Qiao, Y.-Y.; Shen, Z.; Fan, X.-H.; Zhou, Q.-F. Mesogen-Jacketed Liquid Crystalline Polymers with a Polynorbornene Main Chain: Green Synthesis and Phase Behaviors. Macromolecules 2014, 47, 2803-2810. 